\title{
Coulomb Blockade Fluctuations in Strongly Coupled Quantum Dots
}

\author{
S. M. Maurer, S. R. Patel and C. M. Marcus \\ Department of Physics, Stanford University, Stanford, California 94305 \\ C. I. Duruöz and J. S. Harris, Jr. \\ Department of Electrical Engineering, Stanford University, Stanford, California 94305
}

(November 12, 2017)

\begin{abstract}
Quantum fluctuations of Coulomb blockade are investigated as a function of the coupling to reservoirs in semiconductor quantum dots. We use fluctuations in the distance between peaks $\Delta N$ apart to characterize both the amplitude and correlation of peak motion. For strong coupling, peak motion is greatly enhanced at low temperature, but does not show the expected increase in peak-to-peak correlation. These effects can lead to anomalous temperature dependence in the Coulomb valleys, similar to behavior ascribed to Kondo physics.
\end{abstract}

73.23.Hk, 72.23.-b, 73.20.Fz, 73.23.Ad

Typeset using REVTEX 
The Coulomb blockade (CB) of tunneling through a confined island of charge (a quantum dot) at low temperatures provides perhaps the clearest demonstration of the interplay between electron-electron interactions and quantum effects in reduced dimension. For weak tunneling from the dot to electronic reservoirs, CB can be understood as a classical charging effect [1] modified by mesoscopic fluctuations of the coupling of the dot to the leads [2]. As the coupling is increased, transport becomes quite complicated and several effects appear that mix the influence of interaction and quantum interference. These include elastic cotunneling, which shows mesoscopic fluctuations on scales set by the charging energy [3, [4], and coherent enhancement of $\mathrm{CB}$ that is sensitive to time-reversal symmetry breaking [0,6]. At very low temperatures and strong tunneling, Kondo resonances between the spin of the dot and the reservoirs further modify transport [7], as observed in recent experiments [8,9].

In this Letter, we report measurements of mesoscopic fluctuations of $\mathrm{CB}$ peaks as a function of tunneling strength in symmetrically coupled GaAs quantum dots. Specifically, we examine fluctuations in height and position of $\mathrm{CB}$ peaks as a function of coupling to reservoirs, temperature and dot size. The strong tunneling regime has been investigated previously [4, 8, 9, [10,[1], though fluctuations of peak position have not been addressed. Peak position statistics are of particular interest because one expects quantum fluctuations to be greatly enhanced in the strong tunneling regime as coherence and charging effects mix; one also expects a corresponding increase in the number of peaks over which fluctuations are correlated [5.12]. Both of these effects arise from the fact that for strong tunneling a large number $E_{c} / \Delta$ of quantum levels make comparable contributions to conductance, where $E_{c}$ is the charging energy and $\Delta$ the mean quantum level spacing. This contrasts the weaktunneling regime, where low-temperature transport is mediated by tunneling through the ground state only.

Our picture of strong tunneling in semiconductor quantum dots is based on recent theory [5.12.13] that extends inelastic cotunneling [14] and methods applicable to metallic grains [15] by including elastic contributions. Refs. [5, 12] specifically consider dots with asymmetric lead transmissions $T_{1} \ll T_{2} \leq 1$ and so may not be fully applicable here. Also, since neither 
mesoscopic fluctuations of coupling strengths, nor Kondo-type resonances [7], nor changes in the energy spectrum upon addition of electrons [16] are included, we do not anticipate complete agreement between experiment and theory.

We find experimentally that CB peak motion is enhanced for strong tunneling (see Fig. 1), as predicted, but that the number of correlated peaks is not enhanced by the expected factor of $\sim E_{c} / \Delta$. This situation leads to unanticipated experimental consequences, including anomalous (reversed) temperature dependence of $\mathrm{CB}$ valleys, an effect that has been identified as a signature of the Kondo effect in smaller devices [8,9].

We introduce a measure of peak fluctuations for an ensemble of CB peaks as the standard deviation of the distance between peaks $\Delta N$ apart,

$$
\sigma_{p}(\Delta N)=\left\langle\left\langle\left(\tilde{V}_{N}-\tilde{V}_{N+\Delta N}\right)^{2}\right\rangle_{B}^{1 / 2}\right\rangle_{N}
$$

where $\tilde{V}_{N}=V_{N}-\left\langle V_{N}\right\rangle_{B}$ is the position in gate voltage $V_{N}$ of the maximum conductance of the $N^{\text {th }}$ peak minus its average over magnetic field, $\left\langle V_{N}\right\rangle_{B}$. The quantity $\sigma_{p}(\Delta N)$ generalizes the well-studied peak spacing fluctuations, $\sigma_{p}(1)$ [17, 18, 19], and, unlike fluctuations of the peak position itself, is not very sensitive to experimental drift [20]. Noting that $\sigma_{p}(0)=0$, correlated peak motion appears as a reduction in $\sigma_{p}(\Delta N)$ for small $\Delta N$ whose width gives a measure of the number of correlated peaks. For larger $\Delta N$, in chaotic or disordered dots one would naively expect $\sigma_{p}(\Delta N) \propto \log (\Delta N)$ as long as $\Delta N<E_{T} / \Delta$, where $E_{T} \sim h v_{F} / \sqrt{A}$ is the Thouless energy 21] for a ballistic dot of area $A$. Experimentally, we find $\sigma_{p}(\Delta N) \propto$ $\sqrt{\Delta N}$ using large data sets of $50-100$ consecutive peaks in the weak-tunneling regime 22]; that is, we do not observe long-range random-matrix-like correlations, presumably due to changes in the addition spectrum as electrons are added [16]. A related quantity, $\sigma_{g}(\Delta N)$, can be similarly defined as the standard deviation of differences in peak heights (i.e. conductance maxima) for peaks $\Delta N$ apart. Peak heights are not expected to show long-range correlations. Rather, one expects $\sigma_{g}(\Delta N)$ to saturate at $\sqrt{2}$ times the typical height fluctuations of a single peak.

Two scenarios for how strong tunneling might affect $\sigma_{p}(\Delta N)$ are sketched in Figs. 1(d, 
e). In Fig. 1(d), fluctuations are larger for strong tunneling, but the number of correlated peaks does not depend on tunneling strength. This reflects a picture in which a renormalized ratio of $\Delta / E_{c}^{*}$ leads to larger peak motion, where $E_{c}^{*}$ is an effective charging energy at strong tunneling [23]. Figure 1(e) illustrates an alternative cotunneling picture [5, 12] in which both fluctuations and correlations increase with stronger tunneling. Although we expected Fig. 1(e) to better describe our experiment, we find the data look more like Fig. 1(d).

Measurements are reported for two dots with areas $0.3 \mu \mathrm{m}^{2}$ (small) and $1.0 \mu \mathrm{m}^{2}$ (large) [micrographs in Fig. 2], fabricated using CrAu gates $90 \mathrm{~nm}$ above a two-dimensional electron gas (2DEG) in a GaAs/AlGaAs heterostructure. The 2DEG mean free path exceeds the device size, so transport is ballistic within the dot. Charging energies $E_{c}=300 \mu \mathrm{eV}$ (large dot) and $580 \mu \mathrm{eV}$ (small dot) were measured at weak tunneling from the spacing and thermal width of $\mathrm{CB}$ peaks; quantum level spacings, $\Delta=2 \pi \hbar^{2} / m^{*} A \sim 7 \mu \mathrm{eV}$ (large dot) and $\sim 24 \mu \mathrm{eV}$ (small dot), were estimated from the device area, excluding a depletion width of $\sim 70 \mathrm{~nm}$. Measurements were made in a dilution refrigerator using an ac voltage bias of $5 \mu \mathrm{V}$ at $13 \mathrm{~Hz}$. The temperature $T$ refers to the electron temperature, measured from weak-tunneling peak widths.

Figure 1 shows typical $\mathrm{CB}$ peaks as a function of gate voltage $V_{g}$ and magnetic field $B$, illustrating how peak position fluctuations increase with tunneling strength. For weak tunneling, Fig. 1(a), the ratio of fluctuations to average peak spacing is roughly $\sim \Delta / E_{c}$, consistent with theory [24] and some [17] but not all [18] previous experiments. Note, however, that peak spacings gathered over $N$ [17, 18] need not have the same statistics as either peak motions or peak spacings gathered in $B$. As tunneling becomes stronger CB peaks show greatly enhanced motion as a function of $B$, as seen in Fig. 1(b,c). Ensemble statistics of peak position and height fluctuations, gathered from data sets similar to those in Fig. 1, are shown in Fig. 2. Each data set consists of 15 - 20 peaks, with fluctuations gathered over a range of magnetic fields that include many flux quanta through the device ( $\phi_{0} / A \sim 4 m T(\sim 12 m T)$ for the large (small) dot) but remain below the field where the quantum Hall effect appears. 
Three features of these statistics are worth emphasizing. First, peak position fluctuations are seen to increase with stronger tunneling ( Fig. 2(a,c)). This is important given the recent debate concerning the magnitude and origin of fluctuations in peak spacing, $\sigma_{p}(1)$ 17, 18, 19,24, and demonstrate that when comparing fluctuations in different devices, tunneling strength must be taken into account. Second, for the small dot peak position fluctuations actually decrease for the strongest tunneling. This may be related to a similar theoretical result indicating that capacitance fluctuations in the valleys between CB peaks are nonmonotonic in coupling strength [12]. Third, the number of correlated peaks does not depend on tunneling strength. In other words, the curves in Fig. 2 more closely resemble Fig. 1(d) than Fig. 1(e). This is further emphasized in Figs. 2 (b,d,f) which show the same curves, normalized vertically by their values at large $\Delta N$. These scaled curves, denoted $\tilde{\sigma}_{p}(\Delta N)$ and $\tilde{\sigma}_{g}(\Delta N)$, each collapse onto a single curve with the same correlation length. This scaling is not expected theoretically [5, 12], but experimentally appears valid for heights and positions at all measured temperatures, from $45 \mathrm{mK}(85 \mathrm{mK})$ to $300 \mathrm{mK}(400 \mathrm{mK})$ in the small (large) dot.

The absence of correlated peak motion for strong tunneling presumably reflects the fact that the level spectrum of the dot changes as electrons are added [16]. It is known from previous experiments [16,25 that the spectrum near the Fermi surface is rearranged when the number of electrons is changed by roughly $5-8$ for dots of this type and size. Since this number is smaller than $E_{c} / \Delta$, which is 40 (25) for the large (small) dot, these spectral changes rather than elastic cotunneling set the number of correlated peaks.

The temperature dependences of the normalized CB fluctuations, $\tilde{\sigma}_{p}(\Delta N)$ and $\tilde{\sigma}_{g}(\Delta N)$, averaged over different tunneling strengths (a procedure justified by the scaling in Fig. 2) are shown in Fig. 3. The large dot shows the expected increased correlation at higher temperatures $\left(k_{B} T>\Delta\right)$. The small dot does not show increased correlations up to several hundred millikelvin, probably due both to the effectively lower temperature (in units of $\Delta$ ), and to the fact that fewer added electrons are needed to rearrange the spectrum [16]. The unnormalized amplitudes of peak position fluctuations decrease with increasing temperature 
for both dots, consistent with the expected dependence, $\left(k_{B} T / \Delta\right)^{-1 / 2}$ [17].

The fact that peak motion at strong tunneling is enhanced, but correlations are not, can lead to situations in which neighboring CB peaks move quite close together at certain magnetic fields. In this situation, as the temperature is raised, peak fluctuations decrease and the two nearby peaks move apart causing conductance in the valley between them to decrease. Several examples of this are shown in Fig. 4. It is tempting to compare this behavior to recently reported signatures of the Kondo effect in small quantum dots, also measured in the regime of strong tunneling [8.9]. The anomalous temperature dependence of valley conductance that we observe has a roughly logarithmic temperature dependence, as shown in the inset of Fig. 4(b), similar to the Kondo data. However, unlike the even-odd character of the Kondo effect, which leads to a strict alternation of anomalous and normal valleys, the present "peak wandering" effect can appear in adjacent valleys and can switch from one valley to the next by small changes in field (of order $\phi_{0} / A$ ), as seen in Fig. 4. For these anomalous valleys, finite bias measurements also show similarities between the present strong-tunneling data and the Kondo data of Refs. [8,9], including a zero-bias peak and a splitting of the zero bias peak in a perpendicular $B$ field of order $1 T$, consistent with the value $50 \mu V / T$ [9], after averaging peaks and valleys over gate voltage.

While the effects shown in Fig. 4 are not expected within the existing theory of cotunneling [5, 12] it is not clear that they are related to the Kondo effect, despite the similarities discussed above. On the other hand, a spin-dependent strong-tunneling effect similar to a multilevel Kondo resonance [26] could be responsible for the enhanced but uncorrelated peak motion that leads to the present anomalous temperature dependence of valleys, without requiring even-odd alternation. This unexpected behavior demonstrates that the general problem of transport through quantum dots, including interactions, strong tunneling and spin effects continues to provide surprises and challenges.

We thank I. L. Aleiner, S. M. Cronenwett, Y. Gefen, L. I. Glazman, L. P. Kouwenhoven and D. R. Stewart for valuable discussions. We acknowledge support from the ARO 
under DAAH04-95-1-0331 and the NSF-PECASE under DMR 9629180-1 (Marcus Group), and JSEP under DAAH04-94-G-0058 (Harris Group). SMM was supported by the Hertz Foundation. 


\section{REFERENCES}

[1] Single Charge Tunneling, edited by H. Grabert and M. H. Devoret (Plenum, New York, 1992); L. P. Kouwenhoven et al. in Mesoscopic Electron Transport, edited by L. L. Sohn, L. P. Kouwenhoven, and G. Schön (Kluwer, Dordrecht, 1997).

[2] R. A. Jalabert, A. D. Stone, and Y. Alhassid, Phys. Rev. Lett. 68, 3468 (1992); A. M. Chang et al., Phys. Rev. Lett. 76, 1695 (1996); J. A. Folk et al., Phys. Rev. Lett. 76, 1699 (1996).

[3] I. L. Aleiner and L. I. Glazman, Phys. Rev. Lett. 77, 2057 (1996).

[4] S. M. Cronenwett et al., Phys. Rev. Lett. 79, 2312 (1997).

[5] I. L. Aleiner and L. I. Glazman, Phys. Rev. B 57, 9608 (1997).

[6] S. M. Cronenwett et al., Phys. Rev. Lett. 81, 5904 (1998).

[7] L. I. Glazman and M. E. Raikh, JETP Lett. 47, 452 (1988); T. K. Ng and P. A. Lee, Phys. Rev. Lett. 61, 1768 (1988); S. Hershfield, J. H. Davies, J. W. Wilkins, Phys. Rev. Lett. 67, 3720 (1991); Y. Meir, N. S. Wingreen, P. A. Lee, Phys. Rev. Lett. 70, 2601 (1993); N. S. Wingreen and Y. Meir, Phys. Rev. B 49, 11040 (1994); J. König et al., Phys. Rev. B 54, 16820 (1996).

[8] D. Goldhaber-Gordon et al., Nature 391, 156 (1998).

[9] S. M. Cronenwett et al., Science 281, 540 (1998).

[10] T. Heinzel et al., Phys. Rev. B. 52, 16638 (1995).

[11] D. Berman et al., Phys. Rev. Lett. 82, 161 (1998).

[12] A. Kaminski, I. L. Aleiner, L. I. Glazman, Phys. Rev. Lett. 81, 685 (1998).

[13] P. W. Brouwer and I. L. Aleiner, Phys. Rev. Lett. 82390 (1999).

[14] K. Flensberg, Phys. Rev. B 48, 11156 (1993); K. A. Matveev, Phys. Rev. B 51, 1743 
(1995); A. Furusaki and K. A. Matveev, Phys. Rev. Lett. 75, 709 (1995).

[15] D. S. Golubev et al., Phys. Rev. B 56, 15782 (1997).

[16] R. O. Vallejos, C. H. Lewenkopf, and E. R. Mucciolo, Phys. Rev. Lett. 81, 677 (1998); S. R. Patel et al., Phys. Rev. Lett. 81, 5900 (1998).

[17] S. R. Patel et al., Phys. Rev. Lett. 80, 4522 (1998).

[18] U. Sivan et al., Phys. Rev. Lett. 77, 1123 (1996); F. Simmel et al., Europhys. Lett. 38, $123(1997)$.

[19] R. Berkovits, Phys. Rev. Lett. 81, 2128 (1998).

[20] To further reduce experimental noise, all data were taken at positive and negative $B$ values, and a best-fit straight line was subtracted from the peak position to remove small amounts of drift.

[21] M. L. Mehta, Random Matrices (Academic Press, New York, 1967); B. L. Altshuler and B. I. Shklovskii, Sov. Phys. JETP 64127 (1986).

[22] S. R. Patel, S. M. Maurer, C. M. Marcus (unpublished).

[23] L. I. Glazman and K. A. Matveev, Sov. Phys. JETP 71, 1031 (1990); H. Schoeller and G. Schön, Phys. Rev. B 50, 18436 (1994); J. König, H. Schoeller, and G. Schön, Europhys. Lett. 31, 31 (1995); L. W. Molenkamp, K. Flensberg, and M. Kemerink, Phys. Rev. Lett. 75, 4282 (1995); P. Joyez et al. Phys. Rev. Lett. 79, 1349 (1997).

[24] Y. M. Blanter et al., Phys. Rev. Lett. 78, 2449 (1997); R. Berkovits et al., Phys. Rev. B 55, 5297 (1997).

[25] D. R. Stewart et al., Science 278, 1784 (1997).

[26] T. Inoshita, Y. Kuramoto and H. Sakaki, Superlattices and Microstructures 22, 75 (1997). 

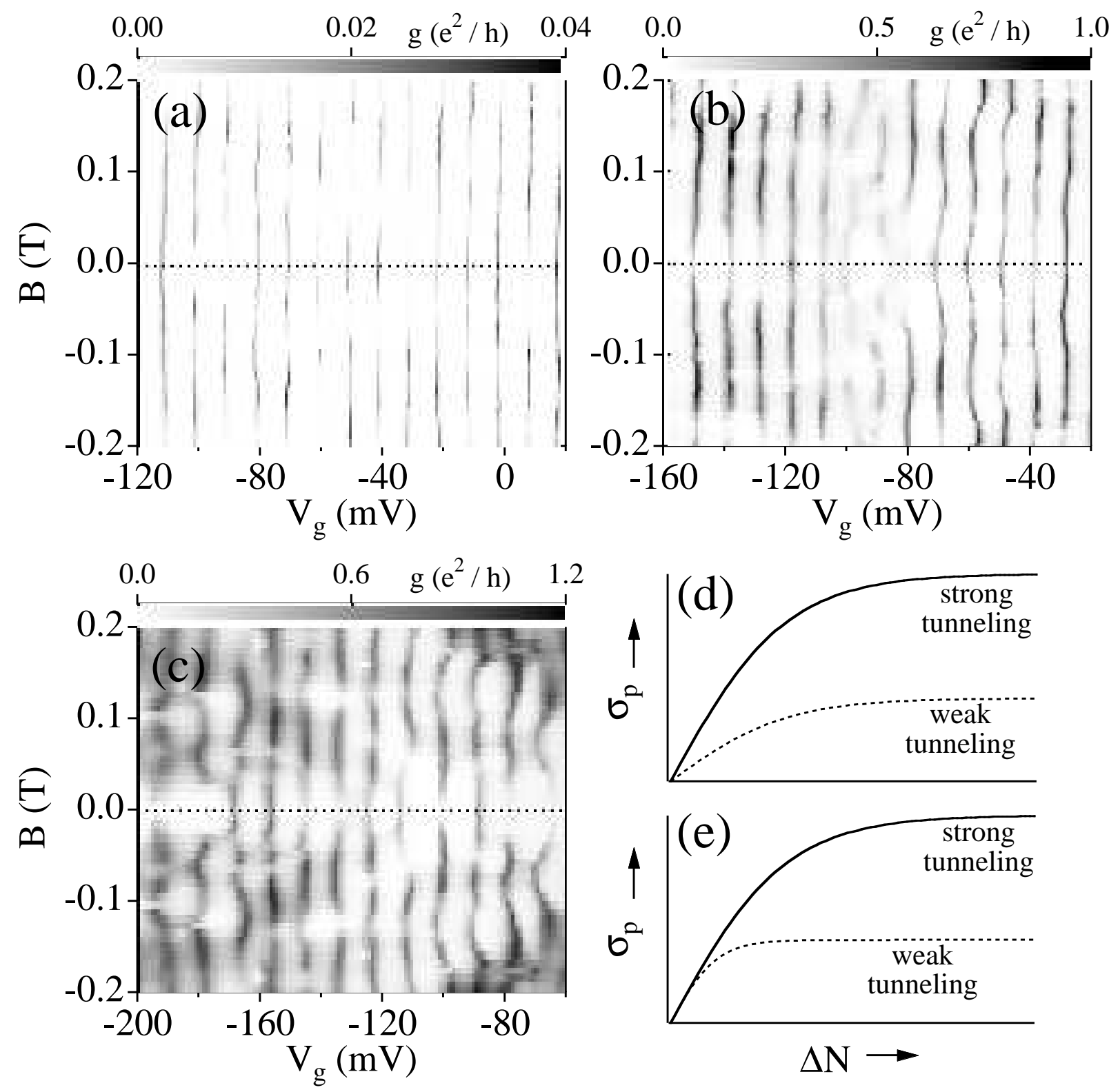

FIG. 1. (a, b, c) Grayscale plots of conductance in the small dot $\left(0.3 \mu \mathrm{m}^{2}\right)$, showing typical CB peak fluctuations for weak and strong tunneling. $\left\langle g_{\max }\right\rangle=0.015$ (a), 0.42 (b) and 0.61 (c). (d,e) Two scenarios for how fluctuations in peak separation $\sigma_{p}(\Delta N)$ grow with the number of peaks $\Delta N$ in the weak and strong tunneling regimes as described in the text. 

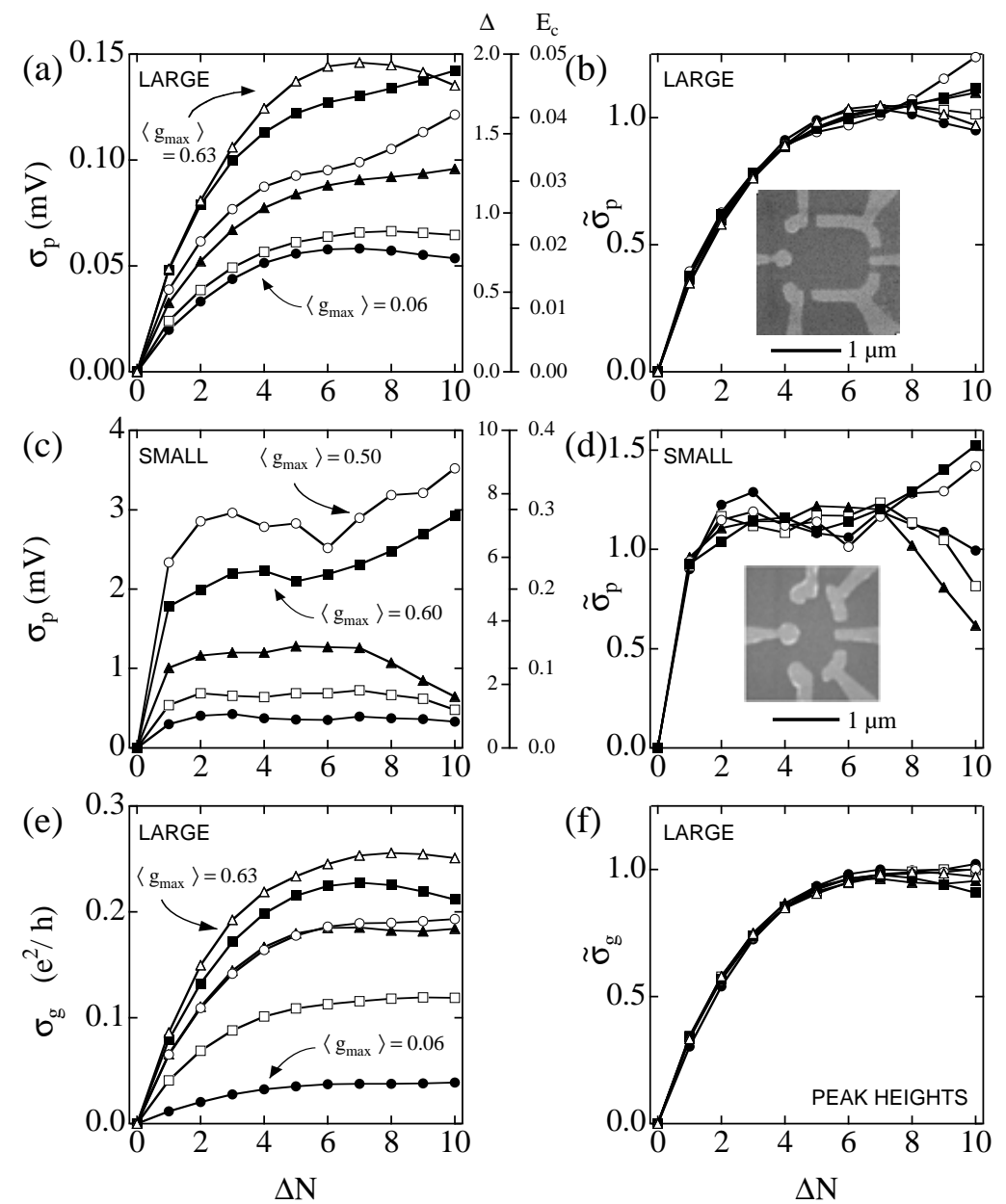

FIG. 2. (a) CB peak fluctuations $\sigma_{p}(\Delta N)$ for the large dot. From bottom (filled circles) to top (open triangles), average peak heights are $0.062,0.28,0.49,0.56,0.60$ and 0.63 . Scales on right show $\sigma_{p}(\Delta N)$ in units of mean level spacing $\Delta$ and charging energy $E_{c}$. (b) Same data as in (a) normalized by its value at large $\Delta N$. Inset: micrograph of large $\left(1.0 \mu \mathrm{m}^{2}\right)$ dot. (c) $\mathrm{CB}$ peak fluctuations $\sigma_{p}(\Delta N)$ for the small dot. Average peak heights are 0.015 (closed circles), 0.32 (open squares), 0.42 (closed triangles), 0.50 (open circles) and 0.60 (closed squares) $e^{2} / h$. Note that fluctuations for $\left\langle g_{\max }\right\rangle=0.50$ are larger than for 0.60. (d) Normalized data from (c). Inset: micrograph of the small $\left(0.3 \mu \mathrm{m}^{2}\right)$ dot. (e) Peak height fluctuations $\sigma_{g}(\Delta N)$ from the data in (a). (f) Normalized peak height fluctuations, $\tilde{\sigma}_{g}(\Delta N)$, from (e), has the same correlation as data in (b). 

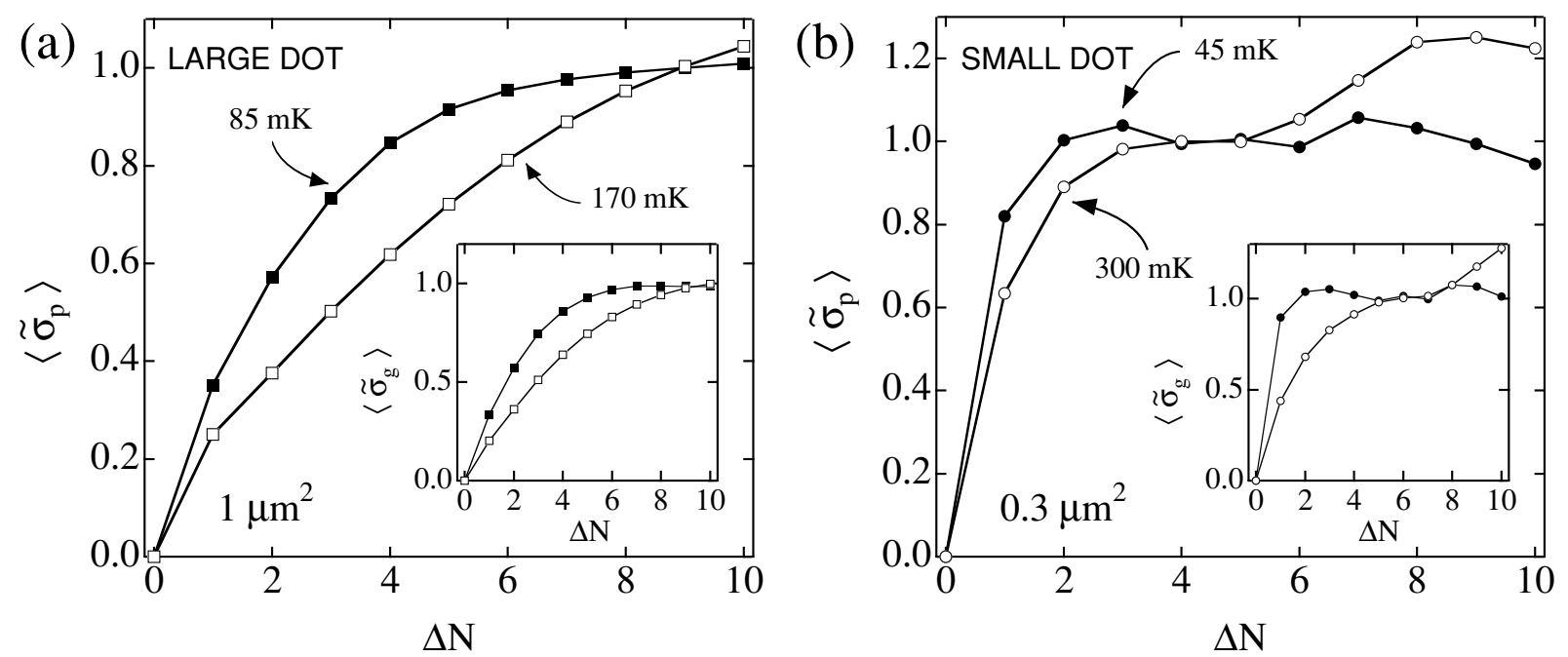

FIG. 3. (a) Temperature dependence of normalized CB peak fluctuations $\tilde{\sigma}_{p}(\Delta N)$ for the large $\left(1.0 \mu \mathrm{m}^{2}\right)$ dot, averaged over the six traces in Fig. 2 (c), at $85 \mathrm{mK}$ (filled squares) and $170 \mathrm{mK}$ (open squares). Inset: same data for peak heights. (b) Same as in (a) for the small $\left(0.3 \mu \mathrm{m}^{2}\right)$ dot at $45 \mathrm{mK}$ (closed circles) and $300 \mathrm{mK}$ (open circles). 

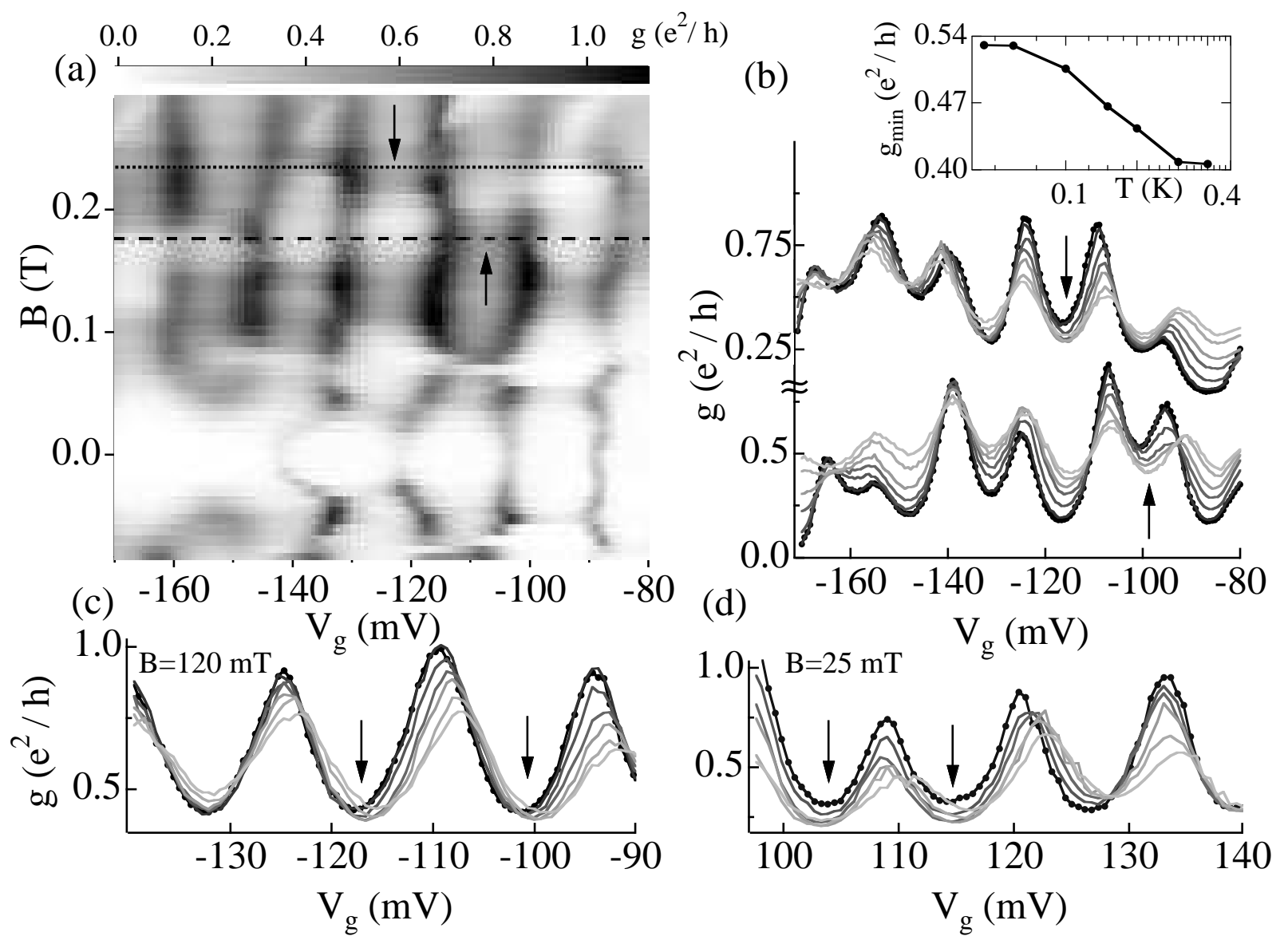

FIG. 4. (a) Conductance (grayscale) as a function of gate voltage $V_{g}$ and magnetic field $B$ for the small $\left(0.3 \mu \mathrm{m}^{2}\right)$ dot at $45 \mathrm{mK}$. (b) Conductances of the two slices in (a) over temperatures from $45 \mathrm{mK}$ (darker, with markers) to $400 \mathrm{mK}$ (lighter). Adjacent valleys with anomalous temperature dependence are marked with arrows. Inset: Conductance in the valley marked by the up arrow. (c,d) Two instances of frequently observed anomalous temperature dependence in adjacent valleys at fixed B. 